DOI: 10.17707/AgricultForest.61.4.18

\author{
Naser SABAGHNIA, Mohsen JANMOHAMMADI ${ }^{1}$
}

\title{
A COMPARISON OF NONPARAMETRIC METHODS TO ANALYZE GENOTYPE BY ENVIRONMENT INTERACTION
}

\begin{abstract}
SUMMARY
Analysis of multi-environment trials (METs) of crops for cultivar evaluation and recommendation is an important issue in plant breeding program and evaluating both stability and yield is essential in MET analyses. Fourteen different nonparametric methods used for analyzing genotype $\times$ environment (GE) interaction were compared by applying them to a set of experimental data (11 lentil cultivars in 20 environments). A principal components analysis (PCA) was performed on the rank correlation matrix arising from the application of each method. In this study, high values of Fox and low values of rank-sum were associated with high yield performance, but the other nonparametric methods were not positively correlated with yield. The 14 nonparametric methods can be categorized in three groups: (i) those which are mostly associated with yield level and did not show any association with nonparametric stability statistics; (ii) those in which both yield and stability are considered simultaneously; and (iii) those methods which only indicate stability level. This analysis also separated those methods based on a dynamic concept of stability from those which are based on a static one. The new nonparametric methods (NS1 and NS2) were grouped with the second and third groups. Therefore, the NS2 statistic which indicated the inter-decile range proportion to median is more applicable than the inter-quartile range proportion to median (NS1).
\end{abstract}

Keywords: adaptation, GE interaction, stability, yield performance

\section{INTRODUCTION}

Multi-environment yield trials which are conducted in multiple years and locations are essential to every plant breeding programs to evaluate the improved genotypes different crops. These trials are the most common efforts in agricultural research and different statistical methods for their analysis have received considerable development and discussion (Gauch, 2006). Although, only simple statistical methods would be used if genotypes performed similarly in all environments but in most cases, genotypes and environments interact to each other and indicate genotype by environment (GE) interaction (Sabaghnia et al., 2008b). The GE interaction had an important affect on improvement for

\footnotetext{
${ }^{1}$ Naser Sabaghnia (corresponding author: sabaghnia@maragheh.ac.ir), Mohsen Janmohammadi, Department of Agronomy and Plant Breeding, Faculty of Agriculture, University of Maragheh, IRAN.

Paper presented at the $6^{\text {th }}$ International Scientific Agricultural Symposium "AGROSYM 2015"

Note: The authors declare that they have no conflicts of interest. Authorship Form signed online.
} 
better genotypes buffering and prevents the extrapolation of results of agronomic evaluations from one environment to another (Yan et al., 2007).

Ignoring the GE interaction is problematic when it is larger than the genotype main effect, which is a common issue in multi-environment yield trials or requiring more knowledge of the magnitudes of GE interactions and of the various sources of variation in GE interaction. The analysis of variance estimates the existence, significance and large magnitude of GE interaction but does not describe its importance therefore; statistical strategies had been proposed to analysis of the GE interaction pattern (Gauch et al., 2008; Sabaghnia et al., 2012a). The question, whether the statistical strategy is good to describe the GE interaction, is still discussed among breeders. The first strategy results from the classical analysis of variance model and linear regression model as reviewed by Lin et al. (1986).

The linear regression model has been developed into the additive main effects and multiplicative interaction (AMMI) model (Gauch, 1992) and a review of the theory and applications of this approach versus genotype plus GE interaction (GGE) biplot model (Yan et al., 2000) has been given by Gauch et al., (2008). In contrast, there are nonparametric stability statistics versus the above mentioned nonparametric methods as the third strategy which are largely unaffected by data distribution. The nonparametric stability methods are based on ranks and a special genotype is considered stable if its ranking is constant across test environments. Several nonparametric stability statistics have been developed to explain the GE interaction in multi-environment yield trials (Huehn, 1979; Kang, 1988; Ketata et al., 1989; Fox et al., 1990; Thennarasu, 1995). The objective of this investigation was an estimation of yield stability performance of genotypes in environments via two new nonparametric stability statistics and their comparison with the existent methods.

\section{MATERIAL AND METHODS}

We denote $x i j$ as observed value of the $i$ th genotype in the $j$ th environment $(i=1,2, \ldots, \mathrm{M} ; j=1,2, \ldots, \mathrm{N})$. Let rij be the rank of genotype $i$ in environment $j$ which the lowest value is rank 1 and the highest value is rank of $\mathrm{K}$. The concept of yield stability is practicable; a genotype is the most stable over test environments if its ranks are similar over environments, and so maximum stability $=$ equal ranks over all test environments. The two nonparametric stability statistics as $N S_{i}^{(1)}$ and $N S_{i}^{(2)}$ which are proposed in this paper are:

$$
\begin{aligned}
& N S_{i}^{(1)}=\left(Q_{3}-Q_{1}\right) / M_{d i} \\
& N S_{i}^{(2)}=\left(D_{9}-D_{1}\right) / M_{d i}
\end{aligned}
$$

In the above nonparametric statistics, $Q_{3}-Q_{1}$ is the inter-quartile range, also called the mid-spread or middle fifty, is a nonparametric index of statistical dispersion, being equal to the difference between the upper and lower quartiles. $M_{d i}$ is the median of the genotypes' ranks in the test environments. Also, $D_{9}-D_{1}$ is the inter-decile range is the difference between the first and the ninth deciles. 
The inter-decile range is another nonparametric index of statistical dispersion of the values in a set of data, similar to the inter-quartile range.

The $N S_{i}^{(1)}$ and $N S_{i}^{(2)}$ nonparametric stability statistics which presented here is similar to the nature and concept of environmental coefficient of variation (Francis and Kannenberg, 1978). In other word, the important central tendency of ranks is the median and its related measures of dispersion are inter-quartile or inter-decile range. It would be interesting that compare these nonparametric stability statistics with the environmental coefficient of variation (CV). The CV was designed primarily to exploration in investigation on the physiological basis for yield stability (Francis and Kannenberg, 1978), and was found more practical to characterize genotypes on a group basis rather than individually. However, this procedure and its related concept could be used in the plant breeding because it represents a simple and descriptive tool for investigation of genotypes' stability. Considering these benefits of CV concept, using new nonparametric stability statistics $\left(N S_{i}^{(1)}\right.$ and $N S_{i}^{(2)}$ ) could be useful in GE interaction interpreting and identification of the most stable genotypes especially in nonparametric strategy. The statistics based on yield ranks of genotypes in each environment are expressed as follows (Huehn, 1979):

$$
S_{i}^{(1)}=2 \sum_{j}^{n-1} \sum_{j^{\prime}=j+1}^{n}\left|r_{i j}-r_{i j^{\prime}}\right| /[n(n-1)] \quad S_{i}^{(2)}=\sum_{j=1}^{n}\left(r_{i j}-\bar{r}_{i .}\right)^{2} / \sum_{j=1}^{n}\left|r_{i j}-\bar{r}_{i .}\right| \quad S_{i}^{(3)}=\frac{\sum_{j=1}^{n}\left(r_{i j}-\bar{r}_{i .}\right)^{2}}{\bar{r}_{i .}}
$$

$$
S_{i}^{(4)}=\sqrt{\frac{\sum_{j=1}^{n}\left(r_{i j}-\bar{r}_{i .}\right)^{2}}{n}}
$$

$$
S_{i}^{(5)}=\frac{\sum_{j=1}^{n}\left|r_{i j}-\bar{r}_{i .}\right|}{n}
$$

$$
S_{i}^{(6)}=\frac{\sum_{j=1}^{n}\left|r_{i j}-\bar{r}_{i}\right|}{\bar{r}_{i} .}
$$

Kang's (1988) rank-sum is another nonparametric stability statistics where both the mean yield and Shukla's (1972) stability variance are used as selection criteria. Ketata et al. (1989) proposed plotting mean rank across environments against standard deviation of ranks for all genotypes $\left(\sigma_{r}\right)$ or plotting mean yield across environments against standard deviation of yields for all genotypes ( $\sigma_{m y}$ ). The formula for calculating both standard deviations are expressed as:

$$
\sigma_{r}=\sqrt{\frac{\sum_{j=1}^{n}\left(r_{i j}-\bar{r}_{i .}\right)^{2}}{n-1}} \quad \sigma_{m y}=\sqrt{\frac{\sum_{j=1}^{n}\left(r_{i j}-\bar{x}_{i .}\right)^{2}}{n-1}}
$$

Nonparametric stability statistics as Top, Mid and Low were introduced by Fox et al. (1990) as nonparametric superiority index (SI) using stratified ranking of the genotypes and their ranking was done at each environment separately and the number of environment at which the genotype occurred in the top, middle, and lower third of the ranks was computed.

Thennarasu (1995) proposed the use of the four nonparametric statistics based on the corrected ranks. In other word, the ranks of genotypes in each 
environment were determined according adjusted values $\left(x_{i j}^{*}=x_{i j}-\overline{x_{i}}\right)$. Thennarasu's (1995) nonparametric stability statistics are:

$$
\begin{array}{ll}
N P_{i}^{(1)}=\frac{1}{n} \sum_{J=1}^{n}\left|r^{*}{ }_{i j}-M^{*}{ }_{d i}\right| & N P_{i}^{(2)}=\frac{1}{n}\left[\sum_{J=1}^{n}\left|r^{*}{ }_{i j}-M^{*}{ }_{d i}\right| / M_{d i}\right] \\
N P_{i}^{(3)}=\frac{\sqrt{\sum\left(r^{*}{ }_{i j}-\bar{r}^{*}{ }_{i .}\right)^{2} / n}}{\bar{r}_{i .}} \quad N P_{i}^{(4)}=\frac{2}{n(n-1)}\left[\sum_{j=1}^{n-1} \sum_{\left[j^{\prime}=j+1\right]}^{n}\left|r_{i j}^{*}-r^{*}{ }_{i j}\right| / \bar{r}_{i .}\right]
\end{array}
$$

All of mentioned stability statistics were computed via Microsoft Excel program.

Table 1 . Mean seed yield and nonparametric stability parameters for yield of 11 lentil genotypes evaluated in 20 environments

\begin{tabular}{|l|c|c|c|c|c|c|c|c|c|c|c|c|c|c|c|c|}
\hline & NS1 & NS2 & S1 & S2 & S3 & S4 & S5 & S6 & Top & Mid & Low & RS & NP1 & NP2 & NP3 & NP4 \\
\hline G1 & 1.32 & 0.79 & 3.2 & 7.3 & 26.7 & 2.71 & 2.3 & 8.4 & 30 & 50 & 20 & 6 & 2.30 & 0.38 & 0.48 & 0.58 \\
\hline G2 & 1.48 & 0.63 & 2.7 & 5.5 & 16.8 & 1.94 & 1.5 & 6.9 & 25 & 65 & 10 & 6 & 1.80 & 0.45 & 0.52 & 0.60 \\
\hline G3 & 1.23 & 1.00 & 4.0 & 12.1 & 27.4 & 3.11 & 2.8 & 8.1 & 25 & 30 & 45 & 16 & 3.05 & 0.47 & 0.48 & 0.57 \\
\hline G4 & 1.07 & 0.77 & 3.3 & 8.1 & 22.7 & 2.85 & 2.4 & 6.8 & 15 & 35 & 50 & 18 & 2.25 & 0.30 & 0.39 & 0.46 \\
\hline G5 & 1.97 & 0.86 & 3.3 & 8.0 & 29.5 & 2.43 & 2.0 & 10.0 & 50 & 40 & 10 & 2 & 2.25 & 0.64 & 0.69 & 0.85 \\
\hline G6 & 1.29 & 0.68 & 3.9 & 10.9 & 28.5 & 3.04 & 2.5 & 7.6 & 20 & 40 & 40 & 18 & 2.75 & 0.39 & 0.50 & 0.51 \\
\hline G7 & 1.35 & 0.77 & 3.5 & 8.9 & 23.8 & 2.82 & 2.4 & 7.3 & 15 & 40 & 45 & 9 & 2.60 & 0.40 & 0.44 & 0.53 \\
\hline G8 & 1.52 & 1.15 & 4.1 & 12.3 & 39.4 & 3.48 & 3.0 & 9.9 & 25 & 35 & 40 & 15 & 3.00 & 0.46 & 0.56 & 0.67 \\
\hline G9 & 1.48 & 1.04 & 3.9 & 11.5 & 32.8 & 3.16 & 2.8 & 9.1 & 25 & 30 & 45 & 16 & 2.95 & 0.49 & 0.54 & 0.65 \\
\hline G10 & 1.67 & 1.17 & 4.4 & 15.4 & 49.8 & 3.83 & 3.5 & 11.9 & 40 & 15 & 45 & 13 & 3.30 & 0.55 & 0.65 & 0.74 \\
\hline G11 & 1.32 & 0.80 & 3.9 & 11.4 & 34.0 & 3.34 & 2.9 & 8.98 & 30 & 20 & 50 & 13 & 2.85 & 0.38 & 0.50 & 0.60 \\
\hline
\end{tabular}

The dataset of Sabaghnia et al. (2006) are used in this study. The computed nonparametric stability statistics are given in Table 1. Each one of the nonparametric methods produced a unique genotype ranking which could be comprised by the Spearman's rank correlations between each pair of nonparametric stability parameters. To better understand the relationships among the nonparametric methods, a principal component (PC) analysis based on the rank correlation matrix was performed. When applying the PC analysis, the two first PCs explained 88\% (54 and 34\% by PC1 and PC2, respectively) of the ariance of the original variables. The relationships among the different stability statistics are graphically displayed in a plot of PC1 and PC2 (Fig. 1). In this plot, the PC1 axis mainly distinguishes the methods of FOX and rank sum (RS) from the other methods. Mean seed yield (SY) also groups near these statistics, and we refer to these as Group-I stability measures. The second PC axis separates NP2, NP3, NP4, S6 and NS1 which we will refer to as Group-III, from the other remained stability measures which we will refer to as Group-II (Fig. 1).

\section{RESULTS AND DISCUSSION}

Though several statistical strategies for yield stability analysis have been proposed, they each reflect different aspects of stability nature and maybe no 
single method can adequately explain genotype performance across test environments. The stability of yield is defined as the ability of a genotype to avoid substantial fluctuations in yield over a range of environmental conditions. The different stability models are broadly classified according to Lin et al. (1986) into there are three types of stability known as Type 1, 2 and 3. Lin and Binns (1991) conclude that stability models of Types 1 and 4 are useful for selection, while those of types 2 and 3 are not useful due to non-heritability. According to Becker and Leon (1988), at least two fundamentally different concepts of stability exist, the static and the dynamic. Both concepts are valuable, but their application depends on the trait considered. It seems that static type of stability is not acceptable to most yield breeders, who would prefer a dynamic (agronomic) concept of stability (Becker, 1981; Sabaghnia et al. 2008a). In the agronomic concept of stability, it is not required that the genotypic response to environmental conditions should be equal for all genotypes (Becker and Leon, 1988). For the more important agronomic traits (yield, oil content and etc.), the static concept type of stability would not be beneficial for the farmers and is equivalent to type 1 of stability while the dynamic concept of stability is equivalent to Type 2 of stability (Lin et al. 1986).

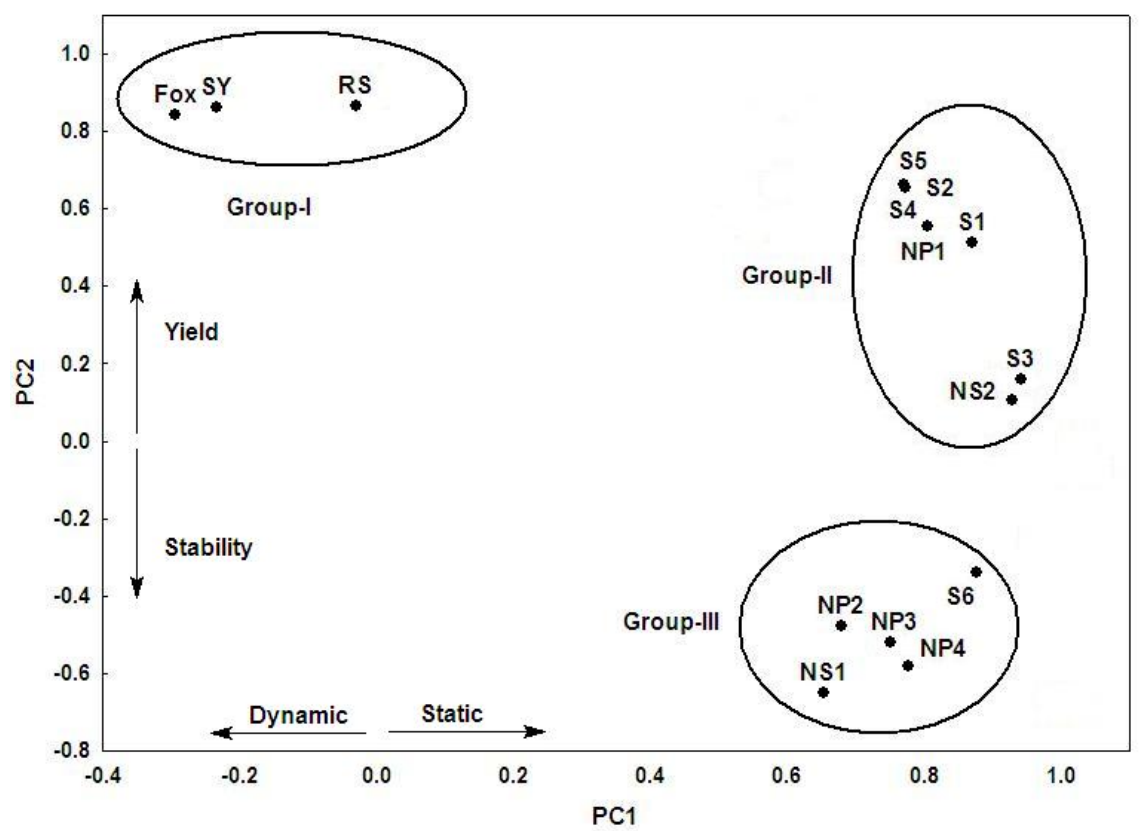

Fig. 1. Principal component analysis (PC1 and PC2) plot of ranks of stability of yield, estimated by nonparametric methods using yield data from 11 lentil genotypes grown in 20 environments and showing interrelationships among these parameters.

It seems that the new nonparametric stability statistics $\left(N S_{i}^{(1)}\right.$ and $\left.N S_{i}^{(2)}\right)$ have similar nature and concept of environmental CV and so benefits from Type 1 of stability while by identification of high mean yield genotype as the most 
stable genotype benefits from dynamic concept of stability. However, for simultaneous selection of mean yield and stability, it is necessary to use mean yield in the formula of each stability statistic. This could be seen in rank-sum (Kang, 1988) as nonparametric stability statistic or desirability index (Hernandez, 1993) as parametric stability statistic. The selection of genotypes for a particular trait depends upon their mean performance and stability statistics. The selected genotypes must have high mean value coupled with stable performance. Most of the nonparametric methods utilized classic stability concept (static or biological concept) for selection of the most favorable genotypes. It seems that there are good poetical in the new introduced nonparametric stability statistics in distinction of favorable genotypes in plant breeding programs. These methods thus provide some flexibility in the hands of plant breeders for simultaneous selection for yield and stability.

\section{CONCLUSIONS}

There are several statistical models for measuring of stability performance and investigation of GE interaction which, they each reflect different aspects of stability and usually no single method can adequately explain genotype performance across environments. The nonparametric stability statistics seem to be useful alternatives to parametric methods (Huehn, 1990b; Yue et al. 1997), although they do not supply information about genotype adaptability. For several reasons, the use of nonparametric stability statistics is preferred. These statistics avoid the bias of outliers and no assumptions are required about the distribution of the observations. Furthermore, these methods are easy to use and to interpret; therefore, estimation of stability seems to be an adequate strategy. Many parametric (univariate and multivariate) and nonparametric statistics of stability have been presented and compared in the literature (Lin et al. 1986; Flores et al. 1998; Sabaghnia et al. 2006). For making practical recommendations, it is necessary to study the relationship among these statistics and compare their powers for different stability approaches.

\section{REFERENCES}

Becker, H. C. and J. Leon. 1988. Stability analysis in plant breeding. Plant Breeding, 101:1-23.

Becker, H. C., 1981. Correlations among some statistical measures of phenotypic stability. Euphytica, 30: 835-840.

Flores, F., Moreno, M. T. and Cubero, J. I. 1998. A comparison of univariate and multivariate methods to analyze $\mathrm{G} \times \mathrm{E}$ interaction. Field Crops Research, 56: 271-286

Fox, P. N., B. Skovmand, B. K. Thompson, H. J. Braun, and R. Cormier. 1990. Yield and adaptation of hexaploid spring triticale. Euphytica, 47: 57-64.

Francis, T. R. and L. W. Kannenberg. 1978. Yield stability studies in short-season maize: I. A descriptive method for grouping genotypes. Canadian Journal of Plant Science, 58: 1029-1034.

Gauch, H. G., 1992. Statistical Analysis of Regional Yield Trials: AMMI Analysis of Factorial Designs. Elsevier, Netherlands, Amsterdam. 
Gauch, H. G., 2006. Statistical analysis of yield trials by AMMI and GGE. Crop Science, 46: $1488-1500$.

Gauch, H. G., H. P. Piepho, and P. Annicchiaricoc. 2008. Statistical analysis of yield trials by AMMI and GGE. Further considerations. Crop Science, 48: 866-889.

Hernández, C. M., J. Crossa, A. Castillo. 1993. The area under the function: an index for selecting desirable genotypes. Theoretical Applied Genetics, 87: 409-415.

Huehn, M., 1979. Beitrage zur erfassung der phanotypischen stabilitat [Contributions to gathering the phenotypic stability]. EDV Medizin und Biologi, 10: 112-117.

Huehn, M., 1990b. Non-parametric measures of phenotypic stability: Part 2. Application. Euphytica, 47: 195-201.

Kang, M. S. and H. N. Pham. 1991. Simultaneous selection for high yielding and stable crop genotypes. Agronomy Journal, 83: 161-165.

Kang, M. S., 1988. A rank-sum method for selecting high-yielding, stable corn genotypes. Cereal Research Communications, 16: 113-115.

Ketata, H., S. K. Yan, and M. Nachit. 1989. Relative consistency performance across environments. Int. Symposium on Physiology and Breeding of Winter Cereals for stressed Mediterranean Environments. Montpellier, July 3-6, 1989.

Lin, C. S. and M. R. Binns. 1991. Genetic properties of four types of stability parameters. Theoretical Applied Genetics, 82: 505-509.

Lin, C. S., M. R. Binns and L. P. Lefkovitch. 1986. Stability analysis: Where do we stand? Crop Science, 26: 894-900.

Sabaghnia, N., H. Dehghani, S.H. Sabaghpour. 2006. Nonparametric methods for interpreting genotype - environment interaction of lentil genotypes. Crop Science, 46: 1100-1106.

Sabaghnia, N., H. Dehghani, S.H. Sabaghpour. 2008a. Graphic analysis of genotype by environment interaction for lentil yield in Iran. Agronomy Journal, 100: 760-764.

Sabaghnia, N., R. Karimizadeh, M. Mohammadi. 2012a. Genotype by environment interaction and stability analysis for grain yield of some lentil genotypes. Žemdirbystè=Agriculture 99: IN PRESS

Sabaghnia, N., S. H. Sabaghpour, H. Dehghani. 2008b. The use of an AMMI model and its parameters to analyse yield stability in multi-environment trials. Journal of Agricultural Science, 146: 571-581.

Shukla, G. K., 1972. Some aspects of partitioning genotype-environmental components of variability. Heredity 28:237-245.

Thennarasu, K., 1995. On certain non-parametric procedures for studying genotypeenvironment interactions and yield stability. Ph.D. thesis. P.J. School, IARI, New Delhi, India.

Yan, W., L. A. Hunt, Q. Sheng, Z. Szlavnics. 2000. Cultivar evaluation and megaenvironment investigation based on GGE biplot. Crop Science, 40: 596-605.

Yan, W., M. S. Kang, B. Ma, S. Woods, P. L. Cornelius. 2007. GGE biplot vs. AMMI analysis of genotype-by-environment data. Crop Science, 47: 643-655.

Yue, G. L., K. L. Roozeboom, W. T. Schapaugh and G. H. Liang. 1997. Evaluation of soybean cultivars using parametric and nonparametric stability estimates. Plant Breeding, 116: 271-275. 